\title{
Diversité Et Variabilité Du Régime Alimentaire Des Éléphants Du Parc National d'Azagny (Sud - Côte d'Ivoire)
}

Kouame Djaha, Enseignant-chercheur

Université Jean Lorougnon Guédé, UFR Environnement, Côte d'Ivoire

Gone Bi Zoro Bertin, Enseignant-chercheur Université Felix Houphouët Boigny, UFR Biosciences, Côte d'Ivoire

Kpangui Kouassi Bruno, Enseignant-chercheur, Koffi Bene Jean Claude, Enseignant-chercheur, Université Jean Lorougnon Guédé, UFR Environnement, Côte d'Ivoire

Koffi Kouao Jean, Enseignant-chercheur, Université Nangui Abrogoua, UFR Sciences de la Nature, Côte d'Ivoire

Vroh Bi Tra Aimé, Enseignant-chercheur Adou Yao Constant Yves, Enseignant-chercheur, Université Felix Houphouët Boigny, UFR Biosciences, Côte d'Ivoire

Abstract

In Côte d'Ivoire, Azagny National Park has a small forest elephants population, described as isolated, self-contained and whose survival depends on the diversity of local biological resources. A study was undertaken to assess the diversity of the plant species consumed and the feeding preference of this large mammal. Pedestrian surveys were carried out in the different biotopes traveled by the elephants during one year, in search of fresh and old traces of nutrition and droppings. As a result of these inventories, six organs of 282 plant species have been identified has consumed by elephant. In this plant species list, three families are the most represented: the Apocynaceae, the Euphorbiaceae and the Rubiaceae. Among the elephant diet, five species appear to be the most consumed: Aframomum melegueta $(1.45 \%)$, Aframomum sceptrum (1.45\%), Sacoglottis gabonensis (1.27\%), Echinochloa pyramidalis (1.2\%) and Albizia adianthifolia (1.2\%). Considering the number and type of organs harvested from plants, the study reveals that they varied from one species to another well. In general, leaves (256 species 90.8\%) and fruits (95 species or 33.7\%) have emerged as the most consumed organs of elephants. In view of this diet importance in elephant survival in the park, it would be important to identify the type of 
species consumed seasonally and to assess their availability in each of the biotopes traveled.

Keywords: National Park of Azagny, Elephant, diet, floristic diversity

\section{Résumé}

En Côte d'Ivoire, le Parc National d'Azagny abrite une population residuelle d'éléphants de forêt, décrite comme isolée, repliée sur elle-même et dont la survie dépend de la diversité des ressources biologiques locales. Une étude a été entreprise pour évaluer la diversité des espèces végétales consommées et la préférence alimentaire de ce grand mammifère. Des prospections pédestres ont été ainsi menées dans les différents biotopes parcourus par les éléphants pendant une année, à la recherche de traces fraîches et anciennes de nutrition et de crottes. A la suite de ces inventaires, six organes de plantes appartenant à 282 espèces végétales ont été identifiés. Dans cette flore, trois familles sont les plus représentées : les Apocynaceae, les Euphorbiaceae et les Rubiaceae. Parmi les espèces qui composent le régime alimentaire de l'éléphant, Aframomum melegueta (1,45\%), Aframomum sceptrum (1,45\%), Sacoglottis gabonensis (1,27\%), Echinochloa pyramidalis $(1,2 \%)$ et Albizia adianthifolia (1,2\%) apparaissent comme les plus consommées. En considérant le nombre et le type d'organes prélevés sur les plantes, l'étude révèle qu'ils variaient d'une espèce à l'autre. D’une manière générale, les feuilles (256 espèces soit 90,8\%) et les fruits (95 espèces soit 33,7\%) sont apparus comme les organes les plus consommés par les éléphants. Vue l'importance de ce régime dans la survie des éléphants dans le parc, il serait important d'identifier le type d'espèces consommées de manière saisonnière et d'évaluer leur disponibilité dans chacun des biotopes parcourus

Mots-clés: Parc National d'Azagny, Eléphant, régime alimentaire, diversité floristique

\section{Introduction}

L'inquiétude concernant la survie de l'éléphant d'Afrique (Loxodonta africana) suite à sa grande perte entre les années 1970 et 1980, en raison du braconnage intense pour l'ivoire, a eu pour conséquence le renforcement des mesures de protection et de conservation de ce grand mammifère. Ces mesures ne peuvent néanmoins se faire qu'en ayant une bonne connaissance de l'animal et ses habitudes. Il s'agit, entre autres, de sa distribution et sa densité, ses mouvements, son comportement, son impact sur les écosystèmes, son régime alimentaire, etc (Kangwana, 1996). Au cours des millénaires passés, plus de 300 espèces d'éléphants se répandirent sur le 
globe, excepté l'Antarctique et l'Australie. A la suite, des lignées entières s'éteignirent, notamment en raison des bouleversements climatiques. Celle des éléphantidés est la dernière à subsister. Il ne subsiste plus que l'éléphant d'Afrique avec deux sous-espèces (Loxodonta africana africana et Loxodonta africana cyclotis) et l'éléphant d'Asie.

En Afrique de l'ouest, la Côte d'Ivoire abrite les deux sous-espèces africaines d'éléphants. Elles sont essentiellement réparties dans les parcs nationaux, les réserves naturelles et les forêts classées (Roth, 1979). Dans le Sud du pays, le Parc National d'Azagny (PNA) abrite encore une population residuelle relictuelle d'éléphants qui trouvent dans ce milieu, un refuge idéal. En effet, 1'expansion des plantations industrielles de palmier à huile et d'Hévéa, a entrainé la dégradation et la réduction progressive des habitats de ce grand mammifère (Lauginie, 2007). Plusieurs sondages effectués auprès des populations riveraines indiquent que leur effectif est d'environ 65 individus (Nandjui, 2005). Ils séjournent dans la zone marécageuse pendant la saison sèche. Au cours de la saison pluvieuse, ils se rendent dans les zones boisées à plus hautes altitudes afin d'échapper à la recrudescence des eaux. Dans ce parc, le rôle de ce grand mammifère dans la régénération de certaines espèces locales a été mis en exergue (Kouamé et al., 2010).

Cette communauté est décrite comme isolée des autres éléphants de la région et repliée sur elle-même. Ainsi la survie de l'éléphant dans le Parc National d'Azagny dépend de la diversité des ressources locales. En effet, les éléphants sont des herbivores stricts, non ruminants (Eboko, 1997). Ils se nourrissent d'herbes, de feuilles, de racines, de fruits, etc. Ce régime alimentaire varie considérablement selon les régions qu'ils parcourent et les périodes de l'année c'est-à-dire des saisons sèches aux saisons pluvieuses tropicales (Soulemane, 2003; Chatelin et al., 2001 ; Mbété et al., 2010, Boukoulou et al., 2012). Bien que de nombreuses études aient été réalisées sur le régime alimentaire de l'éléphant en Côte d'Ivoire (Alexandre, 1961, 1978 ; Soulemane, 2003 ; Theuerkauf et al., 2000), très peu se sont concentrées sur le Parc National d'Azagny (Kouamé et al., 2008). En effet, outre l'étude du volet comportemental,(Nandjui, 2005), la flore constitutive du régime alimentaire de ce grand mammifère est très peu renseignée dans le Parc National d'Azagny dont la formation végétale est constituée d'une mosaïque de végétation. La présente étude s'avère donc nécessaire pour pallier ce manque d'information au niveau de cette aire protégée. L'objet principal de cette étude était d'évaluer la diversité de la flore qui compose le régime alimentaire de l'éléphant dans le PNA. Il s'est agi plus spécifiquement (i) d'identifier la variabilité des espèces végétales consommées par les éléphants et (ii) de déterminer les différents organes végétaux qui font partie de ce régime alimentaire. 


\section{Méthodologie}

Site d'étude

L'étude a été réalisée dans le Parc National d'Azagny (Figure 1), situé dans le sud de la Côte d'Ivoire, entre $5^{\circ} 14^{\prime}$ et $5^{\circ} 31^{\prime}$ de latitude nord et $4^{\circ} 76^{\prime}$ et $5^{\circ} 01^{\prime}$ de longitude ouest. Ce parc couvre une superficie de 21850 ha et abrite une mosaïque de formations végétales très différentes les unes des autres, allant de la savane herbeuse à la forêt dense (Avenard et al., 1971 ; Bouichou, 1978). Le régime hydrique du parc lui confère une spécificité écologique. En effet, le PNA est entouré pour les trois quart de sa superficie, par le complexe fleuve Bandama, canal d'Azagny et lagune Ebrié. Il est inondé à $45 \%$ tout le long de l'année et à $62 \%$ pendant la grande saison des pluies (Roth, 1979).

La faune, caractéristique de celle du domaine forestier guinéen (secteur littoral), s'est enrichie en espèces des milieux lagunaires et d'une avifaune des écosystèmes marécageux (échassiers tels le Héron de Goliath (Ardea goliath), l'Aigle pêcheur (Pandion haliaetus), le Milan noir (Milvus migrans), etc.). Elle s'est adaptée aux conditions marécageuses du milieu, qui lui procurent protection. Les grands mammifères sont représentés par les éléphants, les buffles et l'hippopotame pygmée qui ont trouvé refuge en ce milieu.

Un suivi démographique des éléphants du PNA, indique que leur déplacement se fait généralement suivant les saisons (Lauginie, 1979 ; Nandjui, 2005). Pendant la saison sèche, ils séjournent dans la zone marécageuse. Pendant la saison pluvieuse, ces grands mammifères effectuent une migration dans les zones Nord et boisées du parc, à plus hautes altitudes afin d'échapper à la crue des eaux. Dans cette partie du parc, l'on y rencontre beaucoup de plantations abandonnées qui offrent assez de pâtures (Merz, 1981; EBM, 2000). Cette végétation est beaucoup préférée par les éléphants et est favorable à leur développement. Selon les riverains, les éléphants se seraient réfugiés dans les zones marécageuses pendant les périodes de grandes chasses, ces zones étant pratiquement inaccessibles à l'homme. Avec l'interdiction formelle de la chasse aux éléphants, ces derniers recolonisent la terre ferme qui leur offre beaucoup de variétés de ressources alimentaires. 


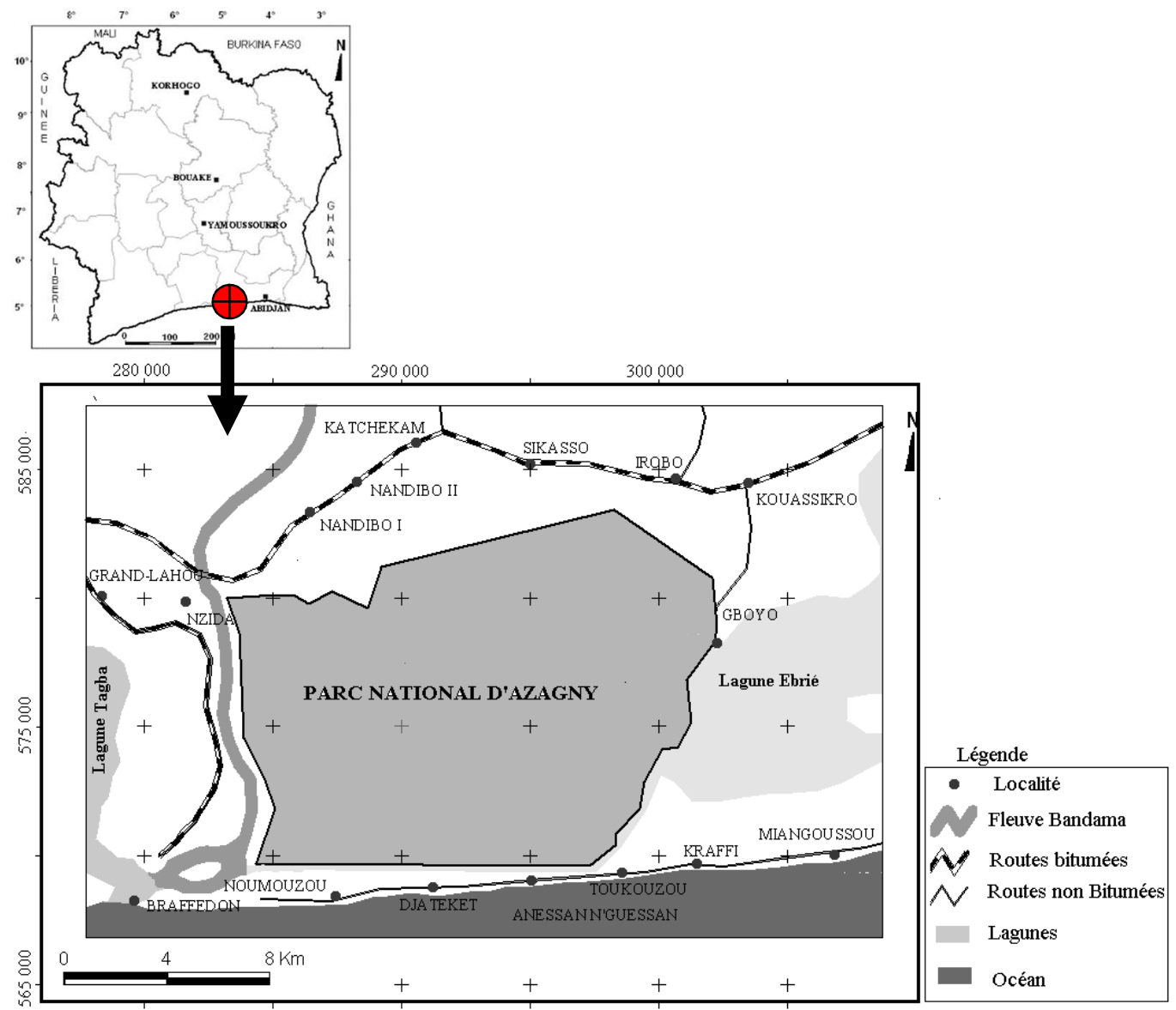

Figure 1 : Cartes de localisation de la zone d'étude (sourc $\square$ : Imag $\square$ CCT-BNETD, 2000)

\section{Collecte des données}

La collecte des données a consisté à suivre la migration des éléphants dans le PNA pendant une année (12 mois) afin d'établir une liste de plantes et des organes consommés à partir d'observations indirectes (Blake, 2002; Mbété et al., 2010). Il a consisté en un suivi quotidien de troupeaux d'éléphants dans les différents biotopes parcourus, à la recherche de traces de nutrition fraîche et/ou ancienne. Dans ces biotopes, des inventaires botaniques ont été effectués à partir de méthode de relevé itinérant de sorte à identifier les espèces végétales et caractériser le type d'organes consommés dans la végétation. Pour chacune des espèces, le nombre d'organes consommés a été noté de manière à pouvoir évaluer les préférences alimentaires et le taux de consommation (Mbété et al., 2010). L'observation indirecte a porté sur des indicateurs de présence d'éléphants dans des anciennes zones de nutrition (crottins, empreintes, arbres et arbustes ébranchés, etc.). Dans les crottes, l'on a procédé à l'identification et à la quantification des graines soit par identification directe sur place ou au 
laboratoire en comparant les graines ingérées à d'autres graines non ingérées, soit par la documentation existante (Kouamé et al., 2008).

\section{Analyse des données}

A partir des données collectées, la richesse de la flore consommée a été déterminée par le dénombrement des espèces recensées sans tenir compte de leur abondance (Aké-Assi, 1984). Les différentes familles correspondantes, ainsi que les types morphologiques (arbre, arbuste, arbrisseau, liane et herbe) et la chorologie ou aire phytogéographique (Guinéo-congolaise, Soudano-zambézienne, etc.) ont été déterminés sur la base des listes d'espèces préétablies par Aké-Assi (2001 ; 2002). Ces paramètres ont permis d'apprécier la richesse et la composition floristique du régime alimentaire de l'éléphant dans le parc.

Les taux de consommation des différentes parties de la plante ont été calculés conformément aux travaux de Kouamé et al., (2010) et Mbété et al. (2010). Des tests de Khi-deux $\left(\chi^{2}\right)$ ont été réalisés pour comparer les différentes proportions d'espèces recensées et les différents taux de consomation calculés. Le logiciel $\mathrm{R}$ a été utilisé pour la réalisation de tous ces tests statistiques

\section{Résultats}

\section{Richesse et diversité de la flore consommée par les éléphants}

Les différentes observations ont permis de recenser 282 espèces végétales, exploitées comme source de nourriture par les éléphants dans le PNA. Elles se répartissent entre 195 genres et 68 familles. 10 espèces de forêt sont les plus consommées que les autres par les éléphants (Figure 2). Parmi elles, Aframomum melegueta (1,45\%), Aframomum sceptrum (1,45\%), Sacoglottis gabonensis (1,27\%), Echinochloa pyramidalis $(1,2 \%)$ et Albizia adianthifolia $(1,2 \%)$ sont les plus importantes. Le régime alimentaire est dominé par 10 familles de plantes. Les plus représentatives sont les Apocynaceae (17 espèces), les Euphorbiaceae (16 espèces) et les Rubiaceae (16 espèces). 


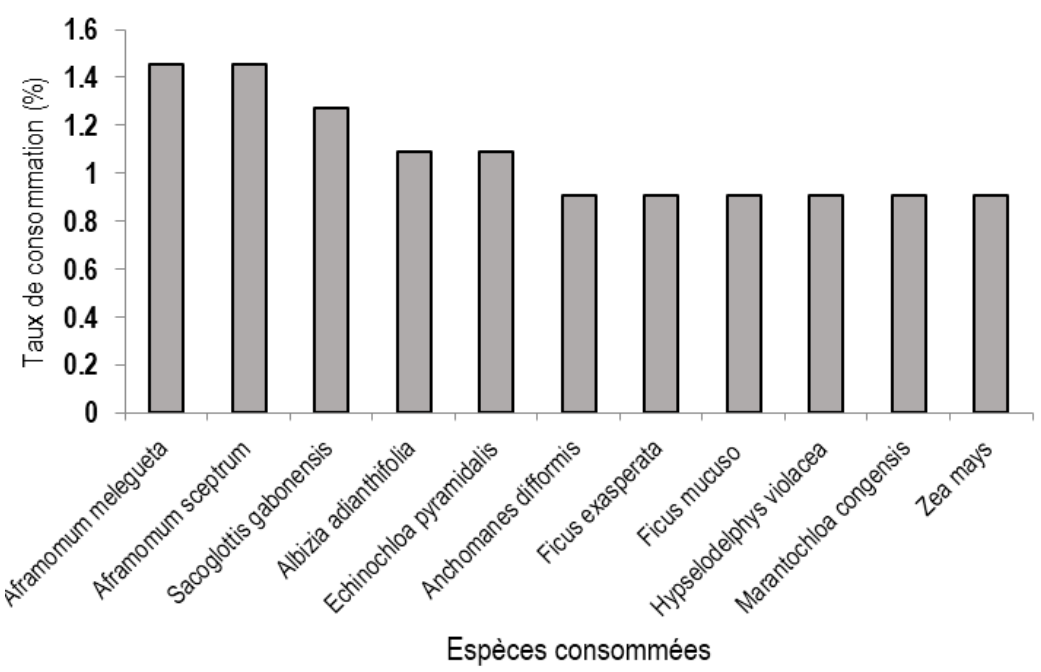

Figure 2 : Histogramme des dix espèces forestières les plus consommées par les éléphants du PNA

\section{Morphologie et aire de distribution de la flore du régime alimentaire}

En considérant les types morphologiques, les espèces arborescentes sont les plus nombreuses dans cette liste floristique (Figure 3). Les arbres et les arbustes sont les plus représentés dans ce régime avec respectivement 109 $(39 \%)$ et 74 espèces (26\%). Les espèces herbacées sont les moins nombreuses avec 19 espèces $(5 \%)$. Les espèces recensées appartiennent à chacune des zones phytogéographiques de la Côte d'Ivoire (Figure 4). Elles sont dominées par celles de la zone forestière (Guinéo-Congolaise) avec 230 espèces végétales $(81 \%)$. Les espèces de régions savanicoles ne sont représentées que par Adansonia digitata et Oryza barthii. A ces espèces locales, s'ajoutent dix espèces introduites dont les plus fréquemment consommées sont: Carica papaya, Psidium guajava, Musa paradisiaca, Musa sapientum, Theobroma cacao et Mangifera indica.

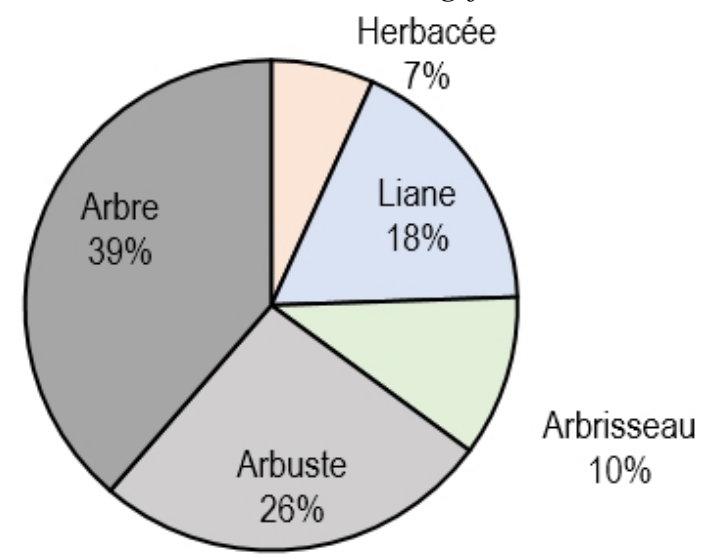

Figure 3 : Spectre morphologique des espèces consommées 


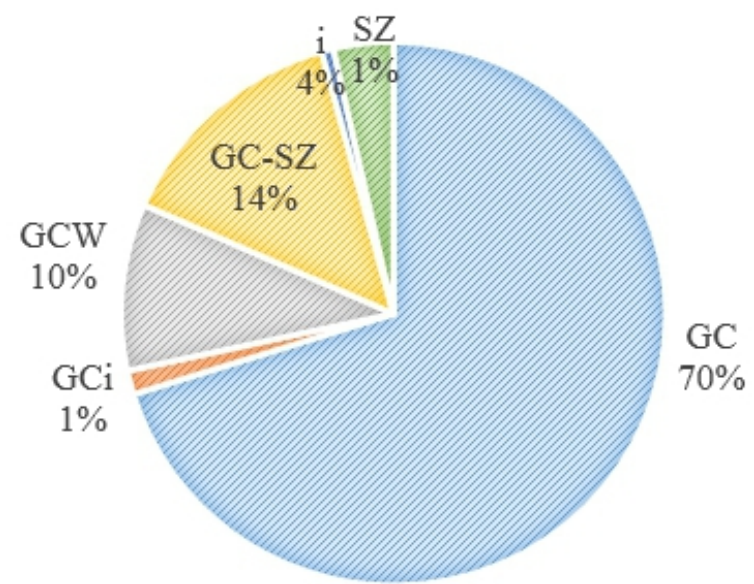

Figure 4 : Spectre chorologique des espèces consommées (GC : taxons de la région GuinéoCongolaise, GCi : taxons endémiques de la Côte d'Ivoire, GCW : taxons endémiques du bloc forestier à l'Ouest du Togo, i : taxons introduites ou cultivés et $\mathrm{SZ}$ : taxons de la région Soudano-Zambézienne)

\section{Diversité des organes consommés}

Le régime alimentaire des éléphants du PNA est constitué de six types d'organes de plantes (Figure 5) pour l'ensemble des 282 espèces végétales consommées. Il est dominé principalement par les feuilles (256 espèces soit $90,8 \%$ ) et les fruits ( 95 espèces soit $33,7 \%$ ) qui constituent les organes les plus appétés. Viennent par la suite, les tiges (37 espèces soit $13,1 \%$ ), les écorces (37 espèces soit 12,4\%), les racines (13 espèces soit $4,6 \%$ ) et les tubercules ( 8 espèces soit $2,8 \%$ ) qui constituent le type d'organe le moins consommé par les éléphants.

En considérant la répartition des types d'organes dans les familles, les Apocynaceae, les Caesalpiniaceae et les Rubiaceae regroupent le plus d'espèces dont les feuilles sont consommées. Au niveau des espèces dont les fruits sont consommés, la famille des Sapotaceae est la plus importante. Pour les autres types d'organes, l'on peut citer les Apocynaceae et les Mimosaceae pour leurs écorces; les Dioscoreaceae et Araceae pour leurs tubercules ; les Marantaceae et Zingiberaceae pour leurs racines ; enfin les Marantaceae et les Apocynaceae pour leurs tiges. 


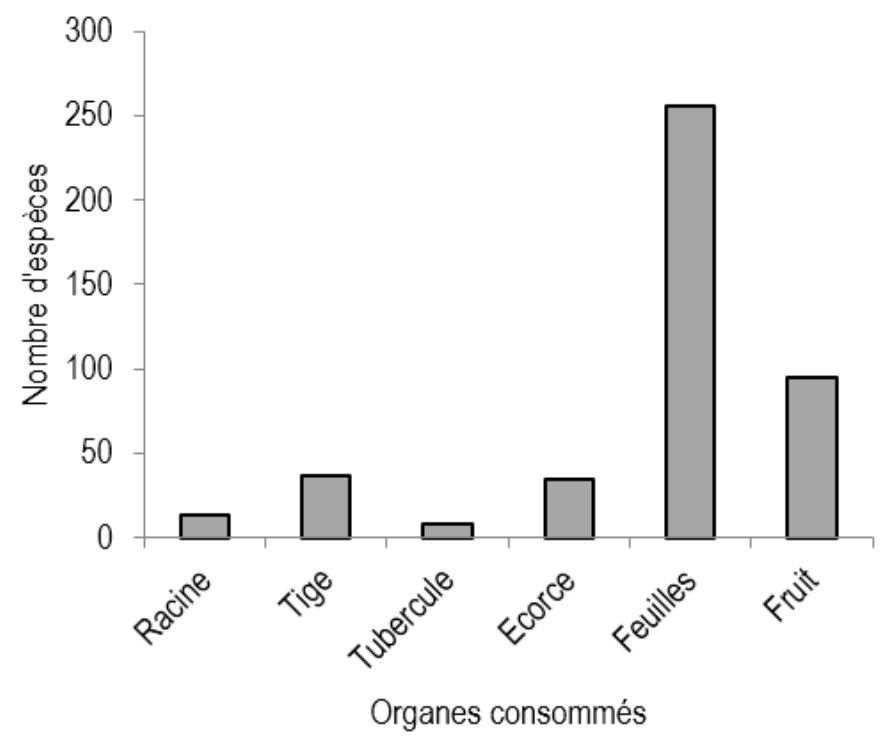

Figure 5 : Histogramme de répartition des organes consommés par les éléphants

\section{Préférence alimentaire des éléphants}

En croisant la liste des espèces et les organes consommés, l'on note que le prélèvement des organes consommés diffère significativement selon les espèces $\left(X^{2}=987.57, P<0,001\right)$. Dix sept groupes d'espèces peuvent être distingués suivant le type d'organe consommé (Figure 6). Les analyses effectuées montrent que les espèces dont les feuilles sont consommées (126 espèces soit $44,7 \%$ ) et celles dont les fruits et feuilles sont simultanément consommés (64 espèces soit $22,7 \%$ ) sont les plus importantes.

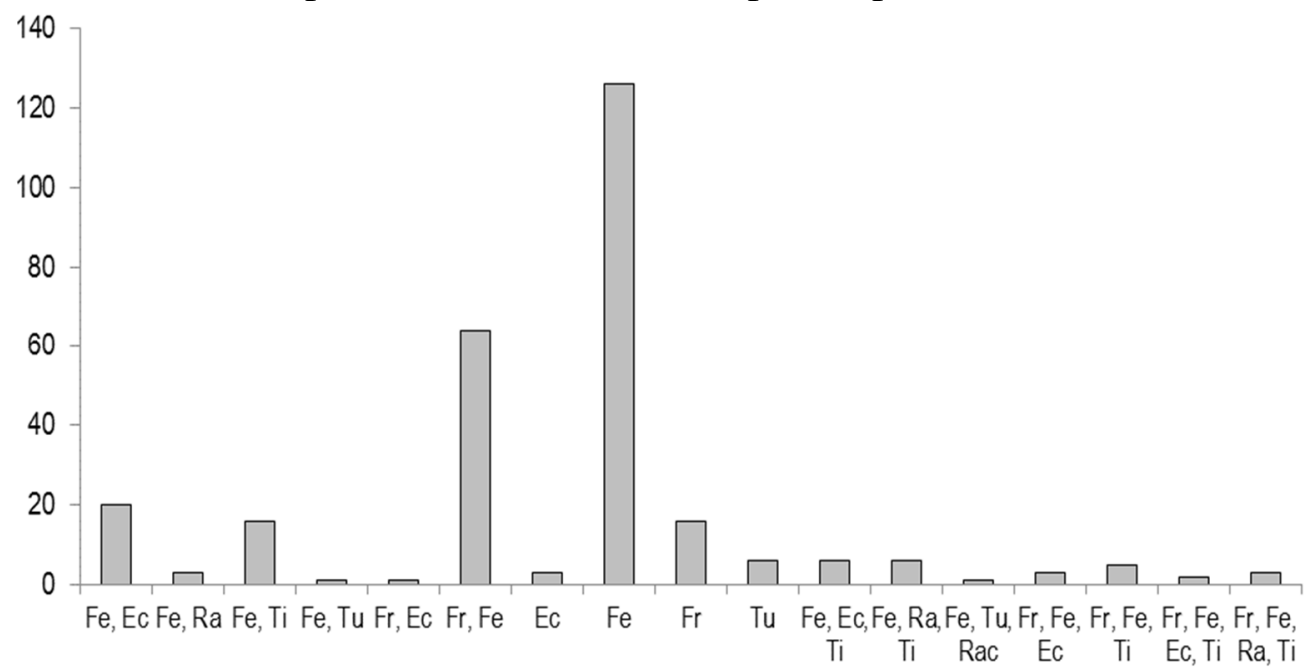

Figure 6 : Histogramme de répartition des groupes d'organes consommés chez les espèces 
L'on peut rassembler ces groupes en quatre grandes catégories d'espèces (Figure 7). La première catégorie rassemble 151 espèces végétales (54\%) chez lesquelles, un seul type d'organe est consommé : la feuille, le fruit, l'écorce ou le tubercule. Dans cette catégorie, les feuilles constituent l'organe le plus consommé avec 126 espèces soit 83,44\% $\left(\mathrm{X}^{2}=277.53, \mathrm{P}<\right.$ 0,001). Pour ces espèces, les familles les plus importantes sont les Apocynaceae (11 espèces), les Euphorbiaceae (11 espèces), les Rubiaceae (10 espèces) et les Caesalpiniaceae ( 8 espèces). Les fruits, avec, 16 espèces (10,6\%), constituent le second type d'organe le plus consommé. Les taux de consommations les plus élevés ont été observés chez Parinari excelsa, Phoenix reclinata, Theobroma cacao, Zea mays et Treculia africana. Les tubercules (six espèces soit 3,97\%) sont consommés uniquement chez Dioscorea spp. Les écorces, enfin, sont consommées uniquement chez trois taxons : Bombax brevicuspe, Entandrophragma angolense et Entandrophragma candollei.

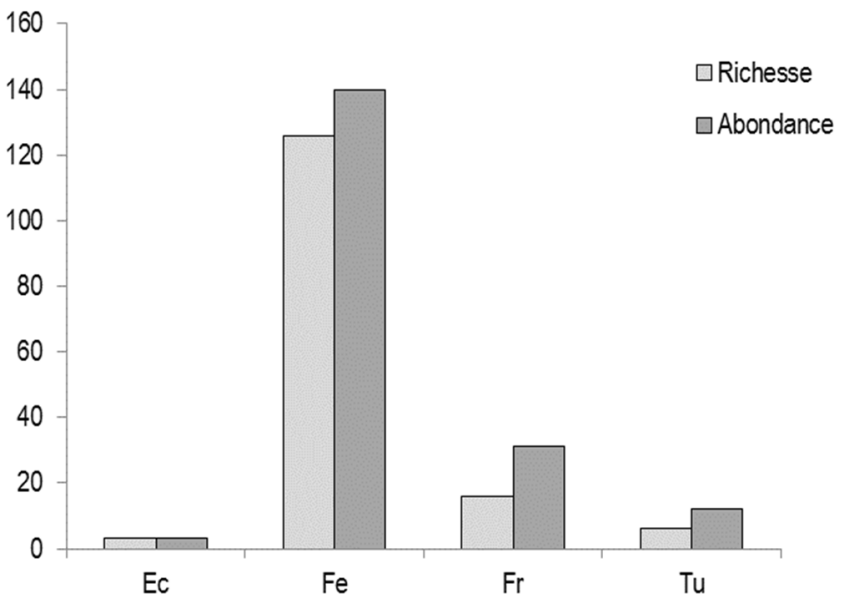

Figure 7 : Histogramme de répartition de la richesse et de l'abondance pour un organe consommé

La seconde catégorie est constituée d'espèces dont deux types d'organes sont consommés sur la plante. Il s'agit de 105 espèces reparties entre six groupes (Figure 8). D'une manière générale, les fruits et/ou les feuilles sont d'abord consommées, puis s'ajoutent les autres types d'organes. Les espèces les plus importantes sont celles qui ont à la fois les feuilles et les fruits consommées $\left(\mathrm{X}^{2}=167.17, \mathrm{P}<0,001\right)$. Elles sont représentées par 64 espèces $(60,95 \%)$ dont les plus importantes sont : Balanites wilsoniana, Canarium schweinfurtii, Carica papaya, Irvingia gabonensis, Psidium guajava, Pycnanthus angolensis et Tieghemella heckelii. Le second groupe le plus important est celui des espèces à feuilles et écorces consommées. Il s'agit de 20 espèces $(19,05 \%)$ dont les plus rencontrées sont: Ficus 
exasperata, Acacia kamerunensis, Bombax buenopozense, Piptadeniastrum africanum, Terminalia superba et le genre Xylopia sp. Le groupe le moins important est celui des espèces à feuilles et tubercule consommées qui est représenté par un seul taxon : Colocasia esculenta.

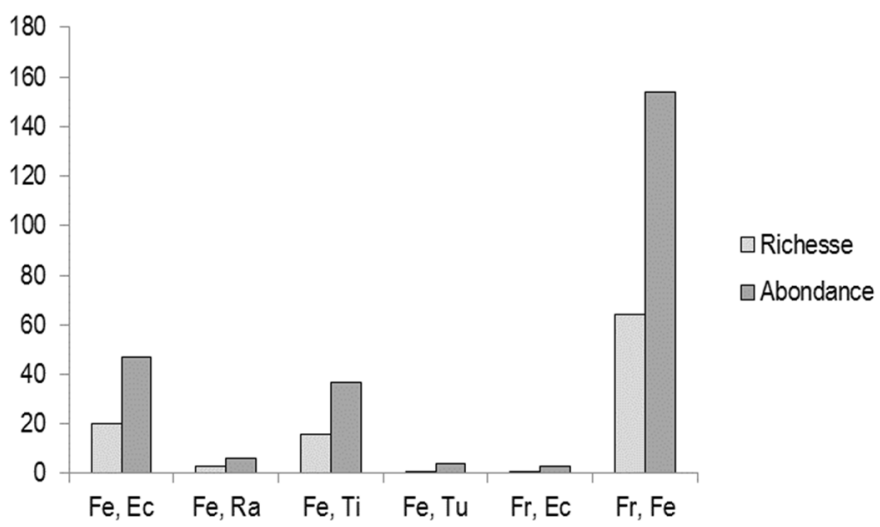

Figure 8 : Histogramme de répartition de la richesse et de l'abondance pour deux organes consommés

La troisième catégorie regroupe des espèces chez lesquelles trois types d'organes sont simultanément consommés par les éléphants (Figure 9). Il s'agit de 21 espèces reparties entre six groupes. Les taux de consommation les plus élevés sont enregistrés chez Hypselodelphys violacea et Marantochloa congensis à la fois pour les feuilles, fruits et tiges ; Ceiba pentandra et Baphia sp. pour les feuilles, écorces et tiges; Anchomanes difformis pour les feuilles, tubercule et racine ; Ficus mисиso et Coula edulis pour les feuilles, écorces et fruits; et Landolphia membranacea pour les feuilles, fruits et tiges.

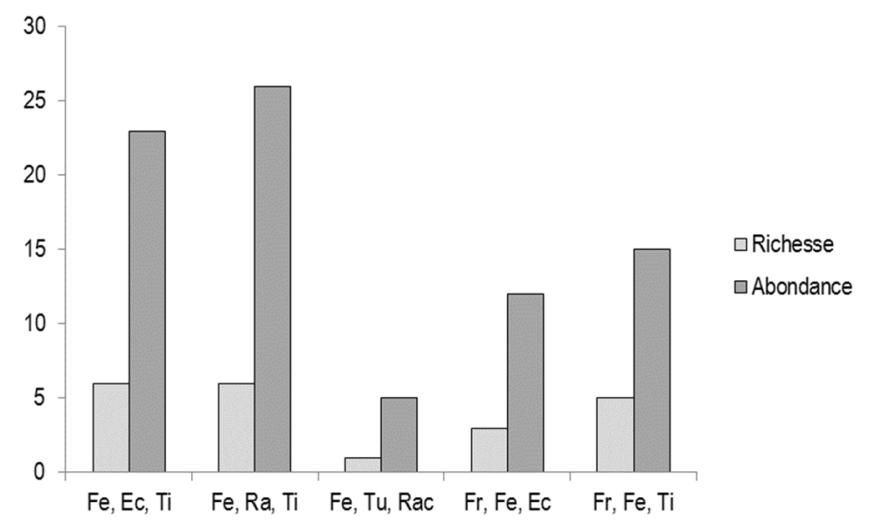

Figure 9 : Histogramme de répartition de la richesse et de l'abondance pour trois organes consomés 
La dernière catégorie rassemble 5 espèces chez lesquelles quatre types d'organes sont consommés. Elles sont reparties entre deux groupes (Figure 10). Il s'agit de: Sacoglottis gabonensis, Albizia adianthifolia, Halopegia azurea pour la feuille, l'écorce, la tige et le fruit ; Aframomum melegueta et Aframomum sceptrum pour la feuille, la racine, la tige et le fruit.

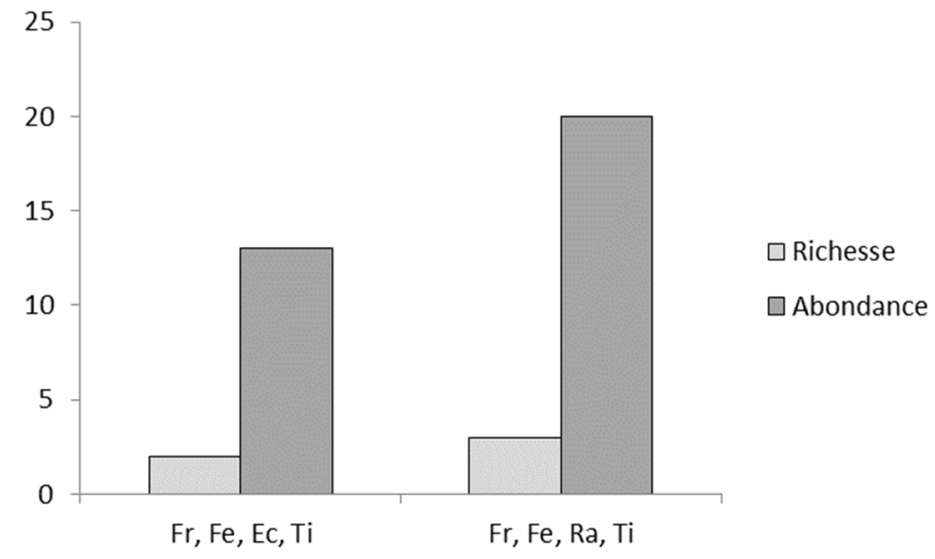

Figure 10 : Histogramme de répartition de la richesse et de l'abondance pour quatre organes consommés

\section{Discussion}

L'étude réalisée a permis de recenser 282 espèces végétales consommées par les éléphants du Parc National d'Azagny. Ce régime est le plus diversifié de celui des éléphants de forêts observé dans les autres Parc Nationaux de la Côte d'Ivoire et de la plupart des pays de la Sous-région (White et al., 1993 ; Theuerkauf et al., 2000 ; Imorou et Sinsin, 2007 ; Mbété et al., 2010). Theuerkauf et al. (2000) ont recensé 147 espèces dans la forêt classée de Bossematié. Dans la réserve de Lopé, au Gabon, 230 espèces consommées ont été recensées par White et al. (1993). En République démocratique du Congo, Mbété et al. (2010) ont recensé 134 espèces de plantes consommées à la périphérie du Parc National Ogooué Lékéti. Cette grande diversité du régime alimentaire pourrait s'expliquer par la diversité de la flore des habitats qui composent le PNA (De Boer et al., 2000 ; Blake, 2002). En effet, dans le PNA, l'on rencontre des savanes côtières, des formations marécageuses, des fourrés, des forêts denses sempervirentes, des forêts secondaires, des forêts à dominance de marécages, des forêts littorales, des forêts de mangrove et des plantations abandonnées (Kouamé et al., 2010). Tous ces biotopes offrent une plus grande variété d'espèces dont la concentration est la plus élevée en Côte d'Ivoire (Kouamé et al., 2008). Pour Blake (2002), il faudrait être prudent lorsque l'on compare les listes 
d'espèces consommées par les éléphants d'un site à l'autre car la diversité pourrait varier en fonction de la durée de suivi des animaux.

Le régime alimentaire des éléphants est polymorphe et est constitué de plusieurs types de plantes et d'organes végétaux. Dans le PNA, les organes d'espèces arborescentes sont les plus consommés par les éléphants. Des observations similaires ont été faites par Blake (2002). Par contre, les travaux de Mbété et al. (2010) et Short (1981) ont montré que les lianes constituaient le groupe d'espèces les plus consommées par les éléphants respectivement en République Démocratique du Congo et au Ghana. Pour certains auteurs, le régime alimentaire des éléphants varie en fonction des saisons (Soulemane, 2003 ; Blake, 2002). Soulemane (2003), dans son étude a montré que dans la Forêt classée du Haut Sassandra (Côte d'Ivoire), l'essentiel de la nourriture des éléphants était constitué de feuilles en saison des pluies et de fruits en saison sèche. En Ouganda, la proportion d'herbes ingérées par les éléphants pendant la saison des pluies, représente $80 \%$ de l'alimentation alors que pendant la saison sèche seulement $40 \%$ d'herbes est ingéré (Field, 1971). Pour cet auteur, au cours de la saison sèche, les herbes (graminées) manquent ou sont souvent de très mauvaise qualité, tandis que les arbres et les buissons, grâce à leurs racines, restent verts bien plus longtemps.

Pour d'autres auteurs, les éléphants s'adaptent et se nourrissent de ce dont ils disposent en abondance (Eboko, 1997 ; Okoumassou et al., 2004 ; Mbété et al., 2010, Boukoulou et al., 2012). L'hypothèse émise par ces auteurs est que l'abondance et la disponibilité de certaines spéculations peuvent faire changer le régime alimentaire des éléphants. Mbété et al. (2010) ont observé que dans l'ensemble de la flore de régime alimentaire des éléphants, une espèce végétale (Hugonia sp 1) très abondante, était la plus consommée dans la zone d'étude. Ils en concluent que l'abondance de Hugonia sp 1 semble orienter le choix des éléphants. Boukoulou et al. (2012) ont montré que les dégâts causées par les éléphants dans les zones périphériques des Parc Nationaux deviennent très importants dès que les cultures arrivent à maturité. Ces spéculations sont généralement représentées par des cultures vivrières (bananiers, manioc, maïs) et des cultures pérennes (cacao). Les résultats obtenus dans cette étude montrent que les éléphants ont des préférences alimentaires qui ne tiennent pas forcement compte de l'abondance d'une espèce particulière. En effet, l'on a observé que le type d'organe prélevé variait d'une espèce à l'autre. Cela suggère qu'ils ont une bonne connaissance de la phénologie des espèces végétales qui composent ce régime alimentaire.

Parmi les différents organes recensés dans cette étude, les feuilles et les fruits sont les plus abondantes. L'importance des feuilles dans le régime alimentaire des éléphants pourrait se justifier par sa disponibilité quasi 
permanente sur toute l'année. Des travaux réalisés sur la nature du régime alimentaire de l'éléphant de forêt ont abouti à des résultats similaires (Alexandre, 1978 ; Blake, 2002, Mbété et al., 2010). Selon les études effectuées par Alexandre (1978) dans le Parc National de la Marahoué, 44 espèces de plantes sont consommées pour leurs feuilles, dont les préférées de l'éléphant sont celles de Solanum verbascifolium, un arbuste au contact irritant, toxique pour l'homme et à odeur forte. Les fruits représentent 24,04\% (95 espèces) du total des espèces consommées par les éléphants dans le PNA. L'importance des fruits dans le régime alimentaire de l'éléphant (Côte d'Ivoire) avait déjà été signalée par de nombreux auteurs en Afrique de l'Ouest (Kouamé et al., 2010). Alexandre (1978) a recensé 42 espèces végétales dans le Parc National de Taï. Theuerkauf et al. (2000) ont identifié 54 espèces de fruits consommés et disséminés par les éléphants de la forêt classée de Bossématié. Au Ghana, Short (1981) dressa une liste de 36 espèces de fruits. White et al. (1993) identifièrent 65 espèces végétales consommées pour leurs fruits par les éléphants de la réserve de Lopé au Gabon. Les fruits consommés par les éléphants sont de grande taille (3 à 5 $\mathrm{cm}$ de diamètre) et appartiennent à des espèces de la strate dominante des forêt. (Kouamé et al., 2010). En tenant compte de la grosseur des fruits, Alexandre (1978) affirme que l'éléphant mange tous les fruits charnus qu'il trouve sur le sol à condition qu'il soit suffisamment gros. Maurois et al. (1997) affirment que ces fruits sont souvent de grande taille, de coloration discrète à maturité (jaune, vert, brun) et présentent une forte odeur. Comme exemples, nous pouvons citer Parinari excelsa, Irvingia gabonensis, Tetrapleura tetraptera, etc. Des auteurs comme Lieberman et al. (1987), Dudley et al. (1992), Yumoto et al. (1995) et Hawthorne et Parren (2000) estiment que pour plusieurs espèces végétales des forêts africaines, telles que Tieghemella heckelii, Balanites wilsoniana et Sacoglottis gabonensis qui ont une importance économique, l'éléphant apparât comme le principal agent de dissémination, en consommant les fruits et en rejetant les graines contenues dans les crottes loin des pieds parents.

Généralement, les graines de fruits consommés par les éléphants sont disséminés intactes. De nombreux fruits couramment consommés par les éléphants montrent une aptitude à la dispersion (loxodontochorie) par ces derniers. Kouamé et al. (2010) ont montré que le taux de germination des graines déféquées par les éléphants a été supérieur à celui des graines non ingérées placées dans les mêmes conditions. L'importance du rôle joué par l'éléphant dans la dissémination des graines dont il consomme les fruits paraît donc indéniable. En effet, de par son régime alimentaire frugivore et ses déplacements journaliers, l'éléphant joue un rôle important dans la biodiversité et dans la régénération des écosystèmes forestiers et savanicoles en contribuant à la dissémination des graines de plusieurs plantes. 
L'importance du rôle disséminateur de l'éléphant pour de très nombreuses espèces végétales, surtout celles à fruits indéhiscents et de grande taille, a été démontrée par plusieurs auteurs (Brahmachary, 1980 ; Short, 1981 ; White, 1994). Ainsi, Alexandre (1978) précise que près de 30\% des graines d'espèces d'arbres de la strate dominante en forêt de Taï sont disséminées par les éléphants dont le crottin joue un rôle favorable sur la germination des graines en accélérant la vitesse de germination et en donnant une remarquable vigueur aux plantules. Selon Adou (2004), certains animaux (Céphalophes, Rongeurs, Oiseaux, etc.) se nourrissent des graines contenues dans les crottes d'éléphant, tandis que d'autres y nichent (Myriapodes, Coléoptères, etc.).

\section{Conclusion}

Cette étude a montré que le régime alimentaire des éléphants du Parc National d'Azagny est très diversifié. Il comprend 282 espèces végétales appartenant principalement aux Apocynaceae Euphorbiaceae et Rubiaceae Parmi les espèces qui composent ce régime, Aframomum melegueta, Aframomum sceptrum Sacoglottis gabonensis Echinochloa pyramidalis et Albizia adianthifolia sont les plus consommées. Dans cette flore, l'on note une dominance d'espèces arborescentes appartenant généralement aux régions forestières. Sur ces différentes espèces recensées, les éléphants prélèvent six types d'organe. Les feuilles et les fruits sont apparus comme les organes les plus consommés par les éléphants. En considérant le nombre et le type d'organe prélevés sur les plantes, l'étude a montré qu'il existe quatre grandes catégories d'espèces dans ce régime. Chez certaines espèces, seul un type d'organe est prélevé. Chez d'autres par contre, le nombre d'organes varie entre deux et quatre. La présente étude ne suffit pas pour appréhender toutes les facettes du régime alimentaire du Parc National d'Azagny. Il serait important d'identifier le type d'espèces consommé par biotope et d'évaluer leur disponibilité afin de prévenir d'éventuels conflits avec les populations locales

\section{References:}

1. Adou, A. (2004). Stratégie de gestion durable des éléphants en Côte d'Ivoire. https://cmsdata.iucn.org/downloads/str_wci2005_fr.pdf

2. Aké-Assi, L. (2001). Flore de la Côte d'Ivoire 1, catalogue, systématique, biogéographie et écologie. Genève, Suisse : Conservatoire et Jardin Botanique de Genève ; Boisseria 57.

3. Aké-Assi, L. (2002). Flore de la Côte d'Ivoire 2, catalogue, systématique, biogéographie et écologie. Genève, Suisse : Conservatoire et Jardin Botanique de Genève ; Boisseria 58. 
4. Alexandre, D. Y. (1978). Le rôle disséminateur des éléphants en forêt de Tai, Côte-d'Ivoire. Société nationale de protection de la nature et d'acclimatation de France, Paris (FRA).

5. Avenard, J. M. (1971). Aspects de la géomorphologie. In Avenard, J.M., Eldin M., Girard G., Sircoulon J., Touchebeuf P., Guillaumet J.L., Adjanohoun E. \& Perraud A. (eds). Le Milieu Naturel de Côte d'Ivoire, pp 7- 70, Mémoire ORSTOM, France.

6. Blake, S. (2002). The Ecology of Forest Elephant Distribution, Ranging and Habitat Use in the Ndoki Forest, Central Africa. Institute of Cell, Animal and Population Biology, Edinburgh,

7. Bouichou, A. (1978). Etude géographique de la réserve d'Azagny et son environnement anthropique. Mémoire de Maîtrise, Université de Paris VIII.79 p.

8. Boukoulou, H., Mbete, P., Mbete, R., Ngokaka, C., Akouango, F., Excelh, B K R \& Vouidibio, J. (2012). Conflit Homme/Éléphant : étude de cas dans le village Miélékouka au Nord du Parc National d'Odzala Kokoua (Congo). Journal of Applied Biosciences 50: 34783484.

9. Brahmachary, R. L. (1980). On the germination of seeds in the dung balls of the African elephant in the Virunga National Park. Rev. Ecol. (Terre Vie), 34: 139-142.

10. Chatelain, C., Kadjo, B., Kone, I., \& Refisch, J. (2001). Relations Faune-Flore dans le Parc National de Taï: une étude bibliographique. Tropenbos-Côte d'Ivoire. $166 \mathrm{p}$.

11. De Boer, W. F., Ntumi, C. P., Correia, A. U. \& Mafuca, J. M. (2000). Diet and distribution of elephant in the Maputo Elephant Reserve, Mozambique. African Journal of Ecology, 38 (3), 188-201.

12. Dudley, J. P., Mensah-Ntiamoah, A.Y. \& Kpelle, D. G. (1992). Forest elephants in a rain forest fragment: preliminary findings from a wildlife conservation project in southern Ghana. African Journal of Ecology, 30: 116-126.

13. EBM (2000). Preliminary report on estimates of elephants numbers from dung counts in the Kakum. Conservation Area. Unpublished report, Elephant biology \& management project 125 conservation international, Cape Coast, Ghana. 53 p.

14. Field, C. R. (1971). Elephant ecology in the Queen Elizabeth National Park, Uganda. African Journal of Ecology, 9 : 99 - 123.

15. Hawthorne, W. D. \& Parren, M. P. E. (2000). How important are forest elephants to the survival of woody plant species in Upper Guinean forests? Journal of Tropical Ecology, 16: 133 - 150.

16. Imorou, S. A. G. \& Sinsin, B. (2007). Les éléphants dans la zone cynégétique de la Djona (Bénin) : régime alimentaire et utilisation de 
l'espace . In : Fournier A., Sinsin B., Mensah G.A., Wangari E. (Eds),Quelles aires protégées pour l'Afrique de l'Ouest ? : conservation de la biodiversité et développement. pp 224-237 IRD, Paris.

17. Kangwana K. (1996). L'étude des éléphants. Série de manuels techniques AWF. 200 p.

18. Kouamé D., Adou Yao C.Y., Kouassi K.E., N'Guessan K.E. \& Akoi K., (2008). Preliminary Floristic Inventory and Diversity in Azagny National Park (Côte d'Ivoire). European Journal of Scientific Research, 23 (4) : 537-547.

19. Kouamé, D., Yao, A.Y.C., Nandjui A. \& N'Guessan, K.E. 2010. Le rôle de l'éléphant dans la germination des graines de Irvingia gabonensis (Irvingiaceae), Balanites wilsoniana (Balanitaceae), Parinari excelsa (Chrysobalanaceae) et Sacoglottis gabonensis (Humiriaceae) en forêt tropicale : cas du Parc National d'Azagny en Côte d'Ivoire. Int. J. Biol. Chem. Sci. 4(5): 1442-1454.

20. Lauginie, F. (2007). Conservation de la nature et aires protégées en Côte d'Ivoire, Edition CEDA/NEI | Abidjan, Côte d'Ivoire. 668 p.

21. Lieberman, D., Lieberman, M. \& Martin, C. (1987). Notes on seeds in elephant dung from Bia National Park, Ghana. Biotropica, 19: 365-369.

22. Maurois, C., Chamberlan, C. \& Marechal, C. (1997). Aperçu du régime alimentaire de l'éléphant de forêt, Loxodonta africana cyclotis, dans le Parc National d'Odzala, République du Congo. Mammalia, 61: 127-130.

23. Mbete, P., Ngokaka C., Akouango F., Inkamba N. \& Pandi K. F. (2010). Contribution to the survey of the food of the Forest Elephant (Loxodonta africana cyclotis) in the Peripheral zone of the National Park Ogooué Lékéti. Pakistan Journal of Nutrition, 9 (12): 11411148.

24. Merz., G. (1981). Recherches sur la biologie de nutrition et les habitats préférés de l'éléphant de forêt, Loxodonta africana cyclotis Matschie, 1900. Mammalia, 45 : 177-185.

25. Nandjui, A. (2005). Étude démographique des populations d'éléphants Loxodonta cyclotis dans le Parc National d'Azagny (Côte d'Ivoire): effectif et distribution spatiale. Mémoire DEA, Université d'Abobo-Adjamé, Abidjan. 41 p. + annexes

26. Okoumassou, K., Durlot, S., Akpamou, K. \& Segniagbeto, H. (2004). Impacts humains sur les aires de distribution et couloirs de migration des éléphants au Togo. Pachyderm, 36: 69-79.

27. Roth M. (1979). Initiation à la Morphologie, la Systématique et la Biologie des Insectes. Mémoire Orstom: Paris 213 p. 
28. Short, J. (1981). Diet and feeding behaviour of the forest elephant. Mammalia, 45: 177-185.

29. Soulemane, O. (2003). Déterminisme des migrations des éléphants de la Forêt classée du Haut-Sassandra, Côte d'Ivoire. Pachyderm 35: 60-70.

30. Theuerkauf, J., Waitkuwait, W. E., Guiro, Y., Ellenberg, H., \& Porembski, S. (2000). Diet of forest elephants and their role in seed dispersal in the Bossematié Forest Reserve, Ivory Coast. Mammalia, 64(4): 447-460.

31. White, L. J. T., Tutin, C.E.G \& Fernandez, M. (1993). Group composition and diet of forest elephants, Loxodonta Africana cyclotis Matschie, 1900, in the Lopé reserve, Gabon. Afr.J. Ecol., 31: 181199.

32. Yumoto T., Maruhashi T., Yamagiwa J. \& Mwanza N. (1995). Seed dispersal by elephant in a tropical rain forest in Kahuzi-Biega National Park, Zaïre. Biotropica, 27: 526-530. 